\title{
The enigma of cannabis use in spinal cord injury
}

\author{
Richard Acland ${ }^{1}$
}

Received: 20 May 2018 / Accepted: 26 May 2018

(c) International Spinal Cord Society 2018

\begin{abstract}
Cannabis use in medicine continues to confound practitioners. There is confusing interpretation of the efficacy and adverse event data, highlighting the complexity of this unique plant. Cannabis may have a neuroprotective role in SCI.
\end{abstract}

\section{Article}

We are provided with two diametrically opposed viewpoints on the medicinal use of cannabis $[1,2]$. Such is the polarizing effect this subject has on practitioners in general.

It is stated that $\sim 25 \%$ of spinal cord injured (SCI) persons report being users of cannabis for therapeutic purposes. This figure is significant, considering that they may well be living in an environment where marijuana remains illegal.

So does cannabis have a place in SCI care?

We note that cannabis pharmacology is complicated. The composition of the plant cannabis sativa is complex: consisting of hundreds of cannabinoids (the most common being (BD and THC) as well as terpenes, flavinoids, and phenoids. All these substances have definite physiological effects: the sum of the contributions is called the entourage effect. The cannabis effect, metaphorically, is like the sound produced by an orchestra; there are many components that are delivered to produce "the symphony." What is soothing to some may not be to others.

Berliner states that "it is a highly heterogenetic compound" and hence "with the lack of standardization and reproducibility of these (products), it is impossible to generalize study outcomes to the products available to marijuana users."

As health practitioners, we do remain polarized, preferring to interpret the data according to our bias. The data sadly is often not robust because of the difficulty of conducting reputable randomized research in patients with

Richard Acland

r.n.acland@xtra.co.nz

1 Rehabilitation Medicine Burwood Academy, Mairehau Road, Christchurch 8140, New Zealand symptom complexes that are challenging to measure precisely.

Pain, in particular, is extremely challenging. Graves notes the disturbingly high incidence of pain in SCI and the lack of effective therapies.

Reference has been made to the National Academies of Science, Engineering and Medicine Report, January 2017 [3]. I encourage all to read this well considered document which highlights the level of evidence for the use of cannabis in the management of a number of symptom complexes; many of which are often observed in SCI persons.

Is there a specific cannabis effect in SCI?

The two authors discuss its use in pain of SCI origin and Berliner notes limited efficacy in spasticity. Neither mentions other possible uses.

Endogenous activation of the endocannabinoid system occurs after SCI. Some researches are considering the possible neuroprotective role that cannabis might possess and as a consequence possibly affecting outcome [4].

Cannabis has a well recognized adverse event profile. Unlike opioids, acetaminophen and alcohol, overdose on its own does not result in death. I wonder whether its specific classification for "medicinal" or "recreational" use is really appropriate. Cannabis may be too difficult to prescribe under our pharmacological prescription paradigm. Maybe its use should be considered as "for health wellbeing purposes" and remove the stigma of "recreational."

In summary, cannabis challenges us in its complex effects within the Central Nervous System. Practitioners remain polarized as we await greater therapeutic outcome certainty.

\section{Compliance with ethical standards}

Conflict of interest The authors declare that they have no conflict of interest. 


\section{References}

1. Berliner J, Collins K, Coker J. Cannabis conundrum. Spinal Cord Ser and Cases. 2018. https://doi.org/10.1038/s41394-018-0097-0.

2. Graves DE. Cannabis shenanigans: advocating for the restoration of an effective treatment of pain following spinal cord injury. Spinal Cord Ser and Cases. 2018. https://doi.org/10.1038/s41394-0180096-1.
3. National Academies of Sciences E, Medicine.. The health effects odf cannabis and cannabinoids: the current state of evidence \& recommendations for research. Washington, DC: The National Academies Press; 2017.

4. Arevalo-Martin A, Molina-Holgado E, Garcia-Ovejero D. Cannabinoids to treat spinal cord injury. Prog. Neurpsychopharmacol. BioPsychiatry. 2016;64:190-9. Jan4. 